\title{
GROUP MODELING BUILDING: HOW ENVIRONMENT, CULTURE AND WORK CONDITIONS IMPACT ON THE PROCESS
}

\author{
RINA SADIA \\ Lecturer - Shenkar College of Engineering and Design, Israel.
}

\begin{abstract}
The objective of this paper is to study the impact of culture and work conditions on the process of group modeling building. This process took place in an Israeli factory, in a country of mixture cultures and social backgrounds. The process of building a model involved many participants of different positions in the factory, composing a diverse group with varied inputs. Since the participants were chosen from various levels within the company, they were also from diverse backgrounds in terms of their cultural background, socio-economic status and their work position. These impact their way of thinking and their opinions on the problem. This research applied the existing techniques of group model building process in the Israeli factory, showing that the implementation of this process in real-life differs from theory and requires additional information and tools. This need rises mainly from the dissimilarity of the cultural and social backgrounds of the organization and the workers, differences in the educational level of the employees and their various occupational statuses. These distinct differences suggest revisions and additions to the way this process is performed, such as the need to improve communication skills between participants, the need to establish rules-of-conduct in large groups with diverse backgrounds, and the value of personal conversations in addition to the group process. It is therefore vital for research management teams to acknowledge these differences between group members in order to understand the contradictory information that may come up from different parts of the group during the model-building process, as well as improve the final outcome of the group model building process. Revealing this kind of cultural mixture allows a continuous improvement process of knowledge elicitation through this model building process, thus improving the work of research management teams.

Keywords: culture, feedback loops, group modeling process, knowledge elicitation.
\end{abstract}

\section{INTRODUCTION}

The systems approach distinguishes itself from the more traditional analytical approaches by emphasizing the interactions and connections between the different components of a system. The interactions of the parts become more relevant to understanding the system than understanding the parts. This is the value of systems theory - the whole is more than the sum of its parts. A system is called complex due to the multiplicity of its elements, whether they are natural, technical, economic or social systems. A complex system can be characterized by the interactions between the various parts of the system, which cause internal complex causal structure subjected to feedbacks, and by delayed behavioral reactions, which are counterintuitive and difficult to predict [1].

System dynamics is a methodology, which deals with complex systems. Systems dynamic looks at the same type of problems using the same perspective as in systems thinking. The two fields share the same causal loop mapping techniques, but system dynamics takes an additional step by constructing computer simulation models to confirm that the hypothetical structure can lead to the observed behavior and to test the effects of alternative policies on key variables over time. There are two types of feedback loops: positive loop, which generates a reinforcing and exponential behavior over time, and negative loop, which generates a balancing and equilibrating behavior over time. Interactions between these two types of loops create complex system behaviors of growth and collapse, oscillations and others [1]. The 
modeling process using system dynamics is usually carried out through a group modeling process.

The group modeling process, which is an important process of system dynamics intervention in organizations, is mostly developed by researchers from similar backgrounds and cultures. A research conducted in Israel, a country with a mixture of cultures and social backgrounds, evokes a different experience and approach to the more known group model building (GMB) techniques. The GMB process described above was a part of a larger research exploring the relations between organizational effectiveness, quality culture and employee's health. In order to come up with new insights about GMB, the popular principles of group modeling are reviewed and the firm in which this research took place is subsequently described briefly. In the end, the insights regarding the GMB process and possible improvements are presented, including some modeling process learning critique and conclusions.

The system dynamics group modeling process is primarily based on three fundamental tasks: the first is to elicit knowledge and to reveal the participants' mental models through group participants' interactions. Modelers must elicit knowledge from those that are involved in both operations and/or provide policy decisions in order to develop a useful model that has credibility in the eyes of the managers. This information is then used to develop the model $[2,3]$. The second task requires the use of the system dynamics modeling approach to create the conceptual model of the problem by focusing on key feedback loops. The third task includes the conversion of the conceptual model through mathematical formulations to a computer simulation model that allows for the representation of the behavior over time as well as for policy analysis [2,3]. While each of these three tasks is important and essential for the modeling process, the first task is paramount in determining the quality level of the entire modeling process. The entire group modeling process cannot be better than the information gathered during the initial group sessions. The outcomes of the first task influence the subsequent group modeling processes, and therefore, influence their outcome's quality. This paper deals especially with the first task.

Different situations, cultures and organizational histories can lead to different experiences and needs concerning the application of the GMB process [4]. Different types of interventions exhibit feasibility variation and emphasize the importance of knowing and learning from the wide variety approaches that were used to involve the client in the model building process [5]. Sharing the differences stemming from the differences among situations, backgrounds, cultures and organizational histories is significant for the process of learning and gaining a better insight for a more general effective GMB process. A certain case study, in which systems dynamic was used, provided insights and raised issues concerning the GMB process application [6]. Hereby, we will overview the principles of GMB from the literature, its application to the specific case study. We will share some insights and recommendations concerning the GMB from this experience and will close this research report in an effort to provide specific recommendations for the improvement of the GMB process in certain similar situations.

\section{OVERVIEW OF THE PRINCIPLES OF GROUP MODELING}

When dealing with the GMB process, one typically refers to three key issues: (1) the choice of the group participants (including the number of participants), (2) the procedure for preparing the group sessions and (3) the facilitation of the group sessions.

\subsection{The choice of the group participants}

According to Vennix [7] and Andersen [8], the most important aspect of any modeling process is the selection of the right people who will participate in the model-building endeavor. 
The two main issues concerning the selection of participants in a model-building process are the number of people to involve and how diverse should the group be. The recommendation with respect to these issues is to include those that have the power to act, meaning those that can implement a decision [7]. Vennix [7] also suggests the number of five participants in a GMB as the best size from his experience, but each case needs to be dealt with individually. The larger the size of the group, the more structured the sessions need to be. Andersen [8] emphasizes that it is necessary to include in the group those who have either the support for the effort that is needed, like top management, or those who will carry forward the results of the process. In summary, the suggestion is to choose different stake holders from a variety of backgrounds and culture in order for the modeling to be more efficient and beneficial [8].

\subsection{The procedure of preparing the sessions}

In preparing for a session, there are some considerations that should be taken into account. These include assigning different roles to group members, defining the purpose and the outcome of the session, and planning and carrying out all the logistics.

There is a general agreement in the system dynamics literature about five essential roles in the GMB process [7-9]. These five roles are: the facilitator who needs to pay attention continuously to the group processes and has to focus on the tasks of drawing out tacit knowledge and insights from the group; the modeler/reflector, who reflects on the information he/she sketches and feeds this back to the group; the process coach who usually reflects on his/her findings and provides this information to the facilitator during breaks and helps the facilitator to find ways to keep the group modeling process effective; the recorder, who writes down or sketches the important parts of the group proceedings and the gatekeeper who is the champion of the project and does all the preparatory work [9].

Several guidelines that are useful in planning the agenda are provided [7]. The first stage of the session is the introduction of all participants. The next step is to discuss the agenda. It is important to find out if there is a consensus in the group about the problem that needs to be modeled. The problem definition should be recorded and placed where everybody can see it. In case this is not the first session, reports and conclusions from previous sessions need to be provided. Clarifying what is expected from the group in this session and what outcomes are anticipated is important for participants so as to reduce anxiety. It is important to ensure that there are facilities that enable the recording of what the group is designing, and as a general rule it is advised not to write anything before ensuring that the group agrees on it. It is advisable to have the group cycle back and forth between the problem and the model. This means that there can be silences when people reflect on what has been accomplished and on how the group ought to proceed. Breaks are important to plan ahead. Finally, it is important to record preliminary and final conclusions and insights and leave the participants with a simple but clear picture of the insights, which were gained through the GMB process [7].

Others recommend planning the time so that the needs of those present are met, while time availability and the purpose of the intervention are considered [9]. There should always be room for flexibility. They believe that planning for 15 minutes blocks of time, keeps the group alert, on task, and helps to make progress. They also advise to allocate time for the members of the group so as to develop a group sense. They provide some examples of 'ice breaker' exercises, and recommend working closely with the gatekeeper to engineer the composition of small groups so that cliques are avoided. Their final recommendation is to allocate the last hour or half hour to summarize the whole day's effort in order to build a climax and to leave the conference with a feeling of accomplishment. 


\subsection{The facilitation of the group sessions}

A group facilitator is a person who assists a group in its effort to accomplish its tasks. His concerns are about the process and the structure of the work that is being done rather than the content of the work [10]. In the case of system dynamics, the facilitator needs to have a thorough knowledge of system dynamics and extensive model-building skills in order to be able to ask the right questions during meetings [7]. Some argue that one of the most important tasks of a facilitator is to see and understand the group life (the relationship between the group members, their interdependent and interconnectedness) [10]. By understanding what is going on in the group, the facilitator is able to guide the group in a more productive way by being flexible and accommodating the needs of the group members.

The main responsibility of a facilitator is to help group members. In order to help, one needs to be patient and take enough time to learn the client's problem precisely [7]. Authenticity and integrity are of great importance to build trust between participants and facilitator. A facilitator must be neutral with respect to the content of the discussion otherwise he/she turns to be one of the group members and not an outsider, and thus, his role becomes useless. A facilitator needs to show no preference to participants in order to create a climate of trust [7].

\section{THE GROUP MODELING PROCESS AT THE FIRM}

A field research was conducted at the facility of a firm's subsidiary, which will be called 'the firm' in this paper. Sadia [6] described that the firm produces powdered food blends through sensitive packaging which require high level of quality standards. Concurrently, the firm also needs to maintain high level of effectiveness in order to remain profitable in the global economy where for this line of business, the competition is very intense and remaining profitable is difficult. Further details and information can be found in Sadia's work [6].

The concurrent objectives of maintaining a high level of quality standards, while at the same time minimizing production costs, have obviously created a host of problems for the firm's management. The two conflicting objectives, i.e. reducing costs and facilitating a quality culture, compelled the firm's management to seek a better way to lead in order to attain both objectives simultaneously, rather than compromising one for the other. The requirement of keeping costs low, which leads to temporary employment of some of the employees and low salaries for all of them, brings about a lot of pressure, which in turn creates problems maintaining the level of production quality and lately impacts profitability negatively.

The application of the system dynamics approach to analyze and understand the problems of the firm requires the involvement of all concerned parties within a GMB process. In order to assure that the right individuals are included in the group, it was decided to include only workers with direct connection to production. This meant workers who were included on the team were from the production, maintenance, quality, inventory and operation departments. The group consisted of nine workers including the CEO, the operation manager, the maintenance manager, the inventory manager, a food engineer, the quality manager, a quality assurance employee and two production employees. Hereinafter is an overview of the GMB process [6].

\subsection{Definition of the problem in the firm}

The first phase of building the conceptual model included articulating the problem of the organization as it was perceived by the participants. The articulated problem is described in Figure 1. The model includes five feedback loops. The blue arrow means that both variables 


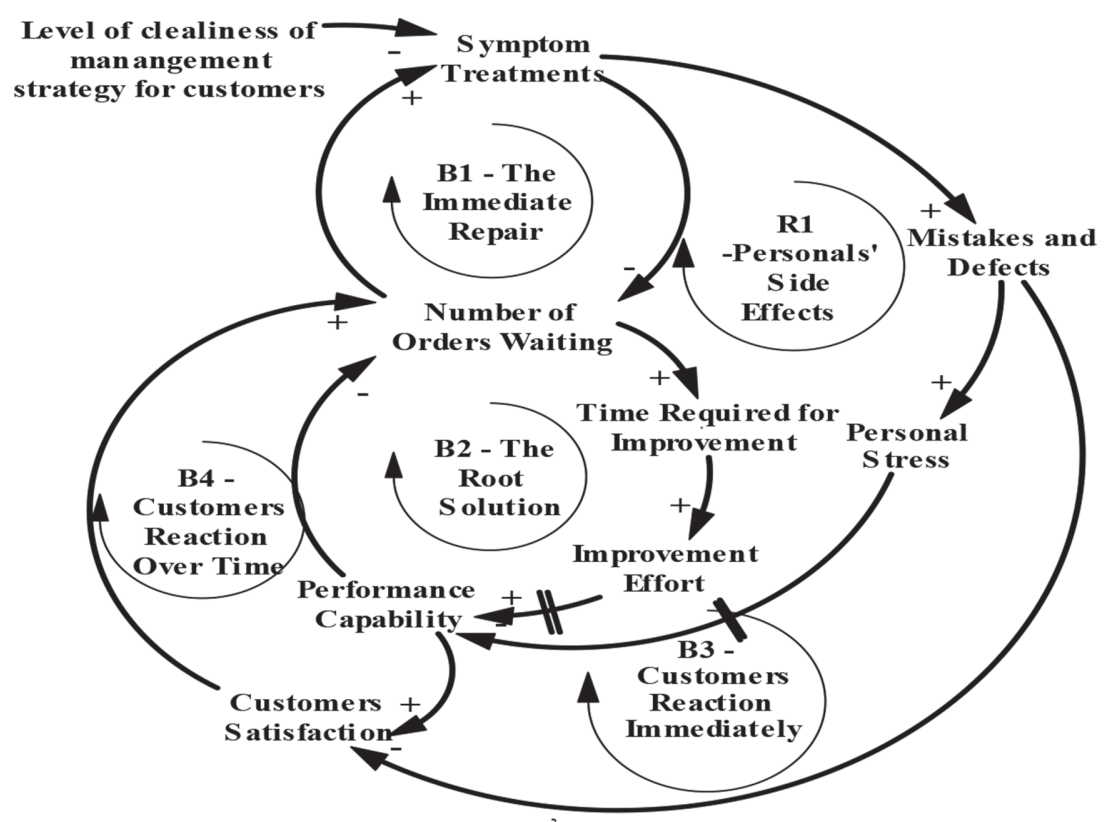

Figure 1: Conceptual model for the problem definition.

behave towards the same direction, either growing or decreasing. The red arrow means that the two variables are contradictory in their behavior. The loop direction, whether being a reinforcing loop (R) or being a balancing loop (B), can be revealed from the arrows' color (an even number of red arrows is a reinforcing feedback loop, whereas an odd number of red arrows is a balancing feedback loop). The model shows the current situation (feedback loop B1), the side effects caused by this behavior (feedback loops R1, B3, B4) and the real solution to the problem (feedback loop B2).

The 'Immediate Repair' feedback loop (B1) was considered as the main issue to deal with. The immediate response to this problem as conceived by the group participants was to push the workers to work harder in less time. This response actually solved the current problem, as described by 'The Immediate Repair' balancing feedback loop. However, such an immediate response caused some side effects.

'Side Effects for Personals' feedback loop (R1) described the side effects on personal health: the more 'Symptom Treatments' involved, the more 'Mistakes and Defects' occurred, causing 'Personal Stress' to increase which led to 'Performance Capability' to decrease and 'Number of Orders Waiting' to increase even more, causing more 'Symptom Treatments' to occur and so on, leading a reinforcing feedback loop.

'Customers Reaction Immediately' feedback loop (B3) was another side effect to 'The Immediate Repair' feedback loop: Increased 'Mistakes and Defects' decreased 'Customers Satisfaction' and therefore fewer orders were handled, decreasing the 'Numbers of Orders Waiting'. 'Customers Satisfaction' decreased also because of the decreasing of the 'Performance Capacity', as described in 'Customers Reaction Over Time' feedback loop (B4).

The root solution to the problem according to the group participants was described through the feedback loop 'The Root Solution' (B2). The accumulating 'Number of Orders Waiting' increased 'Time Required to Improvement' causing an increase to 'Improvement Effort' and 
after a delay - a increase of 'Performance Capacity' causing a decrease of 'Number of Order Waiting'.

From the conceptual model of the problem, one can see that when management's strategy is unclear, the workers tend to look for immediate solutions in order to solve the problem, but on the other hand, it causes side effects. Using a long-term view, the model shows that the investment in improving the process has a balancing feedback loop (B2) affecting the whole system to create more positive situations.

\section{INSIGHTS REGARDING THE GMB AND POSSIBLE IMPROVEMENTS}

The process of articulating the problem and building the model concurrently follows most of the literature guidelines $[7,8,11]$. In this section, the details with respect to these guidelines used will be reviewed. Further, additional elements that were included during the group modeling process will be explained, insights gained will be presented and improvements to the group modeling process will be proposed.

During the beginning stage one has to decide how many participants should be involved in the model building sessions. As mentioned before, the number of five participants in a GMB process is the best size recommended based on accumulated experience [7]; however, each case needs to be dealt with specifically. In this research, the number of participants was chosen to be nine and the sessions had to be highly structured. The group's diversity is advantageous to the model's quality but might create more tension within the group [7]. This conclusion is also reached in this research, as the diversity of the group in this research caused lots of tension and friction within the group, notably with the CEO. Therefore, the facilitator had to be very skillful at conflict resolution when such tensions arose to ensure that the process of building the model did not fall apart. In this case, the participants were chosen from all the company's levels, and as expected when different people in an organization have different interests, these differences are the sole cause of a variety in interpretation [12].

Another stage of the GMB process deals with the preparation of sessions. There were limitations when trying to use the five essential roles [7-9]. In this case, the group participants were not interested in taking on a specific role as mentioned before, therefore the facilitator had to take on some of the roles herself most of the time. In similar cases, where the participants may not be willing to undertake certain roles in the group modeling process, the five essential roles are not usable and not suitable.

The sessions were scheduled according to suggestions [7]; however, there were several important issues that are highlighted as follows:

- Since the group was larger than usually recommended (nine participants) it was deemed useful to hand out rules of conduct that explained their commitments and expected behaviors.

- A feedback form was drafted and critiqued by the group participants. Based on the critique an accepted form was used in all the sessions in order to be able to improve discussions and sessions. The feedback form included questions about the session's contents, its process and personal questions.

- Participation in a group building-model requires a high level of communication. It was important to introduce to the group some concepts on better communication skills [13].

- Since the sessions were scheduled once every three to four weeks, it was deemed necessary to provide a summary of the previous session.

- Specifically for this firm, it was found important to discuss with the group the concept of 'wants' - understanding what someone desires for him and for others that helps her/him to act towards or away from it [13]. Other kinds of problems might need different insights 
and openness (e.g. the difference between feelings and thoughts), since system dynamics by its nature requires many levels of knowledge and understanding.

- In this case, we had to be very careful with the knowledge elicitation process from the group participants. Since the participants in the group were from diverse backgrounds in terms of their culture, economic situation and their work position, they could easily be led by their social condition in their thinking and opinions. Therefore, the process of knowledge elicitation through the group interlocution during the session and on the other hand, knowledge elicitation in a personal conversation brought up contradictory information and called for a continuous improvement process of knowledge elicitation through all this research.

In general, although it is preferable to plan each session in detail, it is important to be flexible during each session, to listen to the participants' intents and desires, since the participants are the facilitator's customers.

The following are recommendations for improving GMB process as the learned lessons from this experience, especially when the group members' background and their personal culture are taken into account:

- If the participants are from a diverse background, culture and education, it is recommended in such cases, to elicit knowledge and information from individuals besides the GMB session.

- The larger the number of group members, the more controlled and prepared the session should be and also less flexible. This is even more important when the level of education is diverse.

- Group modeling is also a learning process both on the personal and the team level, which requires many times raising topics that can help in self-development, improving learning abilities and raising self-awareness, i.e. feelings, wants, thoughts and better communication.

- Guiding the team work is essential and paramount in this endeavor, but no less is the personal connection and acquaintance with each of the group members and other workers, who have a huge influence on achieving data, information, thoughts and opinions in order to better understand the system's behavior.

Last but not least, the key learned issues from this research in which the existing techniques of GMB process were used, brought about some distinct insights concerning the way this process is performed. These insights ensue mainly from the dissimilarity of the culture and social background of the organization and the workers, the difference in educational level of the employees and their occupational status. The more the diversity, the more flexible techniques and facilitation approaches are needed, in order to deal with the special characteristics.

\section{CONCLUSIONS}

In this paper, key methodologies of the GMB process and the way they were performed in the case study were reviewed. The exploration of the differences between the theoretical frameworks of the group modeling process and its actual implementation in a case study indicate that the environment, culture and work conditions have an important impact on the way the process is followed and achieved.

Therefore, attention should be given to the dissimilarity of the culture and social background of the organization and the workers, to the difference in educational level of the employees and their occupational status, while building the conceptual model through the 
steps of the group modeling project. Tools that are offered here for improvement of the GMB process include establishing rules-of-conduct, learning and improving communication skills within the group, constructing the sessions and using various forms in the process and adding personal conversations with the participants. The process of knowledge elicitation has to be used by both the GMB process and personal conversations, in order to reveal underlying information about 'how things are done' in the organization.

\section{REFERENCES}

[1] Sterman, D.J., Business Dynamics: Systems Thinking and Modeling for a Complex World, Irwin-McGraw-Hill: New York, 2000.

[2] Ford, N.D. \& Sterman, D. J., Expert knowledge elicitation to improve formal and mental models. System Dynamics Review, 14(4), pp. 309-340, 1998. https://doi.org/10.1002/(SICI)1099-1727(199824)14:4<309::AID-SDR154>3.0.CO;2-5

[3] Rouwette, E.A., Korzilius, H., Vennix, J.A.M. \& Jacobs, E., Modeling as persuasion: the impact of group model building on attitudes and behavior. System Dynamics Review, 27(1), pp. 1-21, 2011.

[4] Berard, C., Group model building using system dynamics: an analysis of methodological frameworks. The Electronic Journal of Business Research Methods, 8(1), pp. 35-45, 2010.

[5] Rouwette, E.A., Vennix, J.A.M. \& Van Mullekom, T., Group model building effectiveness: a review of assessment studies. System Dynamics Review, 18(1), pp. 5-45, 2002. https://doi.org/10.1002/sdr.229

[6] Sadia, R., A system dynamics approach linking employee health, quality culture and organizational effectiveness. Virginia: Ph.D. Dissertation, Virginia Polytechnic Institute and State University, 2006.

[7] Vennix, J.A.M., Group Model Building, John Wiley \& Sons: New York and New-York, 1996.

[8] Andersen, F.D. \& Richardson, P.G., Scripts for model building. System Dynamics Review, 13(2), pp. 107-130, 1997.

https://doi.org/10.1002/(SICI)1099-1727(199722)13:2<107::AID-SDR120>3.0.CO;2-7

[9] Richardson, P. G. \& Andersen, F. D., Teamwork in group model building. System Dynamics Review, 11(2), pp. 113-137, 1995. https://doi.org/10.1002/sdr.4260110203

[10] Phillips, D.L. \& Phillips, C.M., Facilitated work groups: theory and practice. Journal of Operational Research Society, 44(6), pp. 533-549, 1993.

https://doi.org/10.1057/jors.1993.96

[11] Wolstenholme, F.E., Qualitative vs. quantitative modeling: the evolving balance. Journal of Operational Research Society, 50, pp. 422-428, 1999. https://doi.org/10.1057/palgrave.jors.2600700

[12] Vennix, J.A.M., Group model building: tackling messy problems. System Dynamics Review, 15(4), pp. 379-401, 1999.

https://doi.org/10.1002/(SICI)1099-1727(199924)15:4<379::AID-SDR179>3.0.CO;2-E

[13] Miller, S. \& Miller, A.P., Core Communication, Skills and Processes, Interpersonal Communication Programs, Inc.: Littleton and Co, 1997. 